\title{
Quality of life after breast cancer: Assessment, relevance and effective interventions
}

\section{Lebensqualität nach Brustkrebs: Erfassung, Relevanz und effektive Interventionen}

Authors

Martina Schmidt, Karen Steindorf

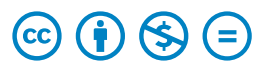

Affiliation

Abteilung Bewegung, Präventionsforschung und Krebs; Deutsches Krebsforschungszentrum (DKFZ) und Nationales Centrum für Tumorerkrankungen (NCT), Heidelberg [Division of Physical Activity, Prevention and Cancer; German Cancer Research Center (DKFZ) and National Center for Tumor Diseases (NCT), Heidelberg]

Key words

breast cancer, quality of life, patient reported outcomes, fatigue, sexual problems

\section{Schlüsselwörter}

Fatigue, sexuelle Probleme, Schlafprobleme, Nebenwirkungen, Langzeitfolgen

Bibliography

DOI https://doi.org/10.1055/a-1030-9792

Senologie 2020; 17: 88-92

(c) Georg Thieme Verlag KG, Stuttgart · New York

ISSN 1611-6453

\section{Correspondence}

Dr. Martina Schmidt

Abteilung Bewegung, Präventionsforschung und Krebs (C110)

Deutsches Krebsforschungszentrum (DKFZ), Im Neuenheimer Feld 581, 69120 Heidelberg

Tel.: ++49/6221/42 2220

m.schmidt@dkfz.de

German version under:

https://doi.org/10.1055/a-1030-9792

\begin{abstract}
Health-related quality of life is of great relevance for breast cancer patients at all stages, both during treatment and in the medium and long term after treatment. Quality of life is becoming increasingly important as an end point in licensing studies for new treatments and in scientific studies comparing different therapies. In addition to a brief global assessment of quality of life, other important patient-reported outcomes should be assessed. Patients with breast cancer often report limitations due to fatigue, sleep problems, sexual and/or climacteric problems, cognitive problems, mental problems and loss of physical performance. Quality of life after breast cancer could probably be further increased if education, screening and treatment of these symptoms were a systematic part of oncological care.
\end{abstract}

\section{ZUSAMMENFASSUNG}

Gesundheitsbezogene Lebensqualität ist für Brustkrebsbetroffene in allen Stadien, sowohl während als auch mittelund langfristig nach Abschluss der Therapie, von großer Relevanz. Bei Zulassungsstudien für neue Behandlungen sowie wissenschaftlichen Studien zum Vergleich verschiedener Therapien gewinnt Lebensqualität als Endpunkt zunehmend an Bedeutung. Neben einer kurzen globalen Einschätzung der Lebensqualität sollten weitere wichtige Patient-Reported Outcomes erhoben werden. Patientinnen mit Brustkrebs berichten häufig über Einschränkungen durch Fatigue, Schlafprobleme, sexuelle bzw. klimakterische Probleme, kognitive Probleme, psychische Probleme und Verlust an körperlicher Leistungsfähigkeit. Die Lebensqualität nach Brustkrebs könnte vermutlich weiter gesteigert werden, wenn Aufklärung, Screening und Behandlung dieser Symptome systematischer Bestandteil der onkologischen Versorgung würden.

\section{Introduction}

Survival after breast cancer has improved markedly in recent years and decades. The average relative 5 - year survival rate of women with breast cancer is approximately $88 \%$ in Germany. The longterm quality of life is therefore of great importance. Quality of life aspects also play an important role during adjuvant therapy, including for therapy compliance and also when deciding between different oncological treatments. Patients want to know how other patients felt during and after the respective treatment. At the advanced stage, when cure is no longer possible, improving or maintaining quality of life is even the primary aim. 
- Table 1 Frequently used generic and cancer-specific quality of life questionnaires.

\begin{tabular}{|c|c|c|c|}
\hline type & \multicolumn{2}{|l|}{ instrument } & items \\
\hline \multirow[t]{4}{*}{ generic } & SF-36 & Medical Outcomes Study Short Form 36 & 36 \\
\hline & EQ-5D-3L & EuroQol Scale (3 possible responses, old version) & 5 \\
\hline & EQ-5D-5L & EuroQol Scale (5 possible responses, since 2009) & 5 \\
\hline & EQ VAS & EuroQol Visual Analogue Scale & 1 \\
\hline \multirow[t]{5}{*}{ cancer-specific } & EORTC QLQ-30 & $\begin{array}{l}\text { European Organisation for Research and Treatment of Cancer Quality of Life - Core } \\
\text { Questionnaire }\end{array}$ & 30 \\
\hline & EORTC QLQ-BR23 & Additional breast cancer-specific module (old version) & 45 \\
\hline & EORTC QLQ-BR45 & Additional breast cancer-specific module (since 2018) & 45 \\
\hline & FACT-G & Functional Assessment of Cancer Treatment - General & 27 \\
\hline & FACT-B & Functional Assessment of Cancer Treatment - Breast & 37 \\
\hline
\end{tabular}

The term 'Quality of Life (QOL)' first appeared in the medical literature in the $1960 \mathrm{~s}$. However, it took a good 30 years before quality of life was recognised as a relevant end point in clinical studies. More recently, subjectively perceived patient-reported outcomes (PROs), including self-reported health-related quality of life, have gained greatly in importance in oncological studies. The use of PROs in licensing studies is increasingly required by the American Food and Drug Administration (FDA) and the European Medicines Agency (EMA) to record symptoms, impairments and side effects of the treatment [1]. It is essential to record quality of life and symptom burden from the patients' point of view as physicians' assessments often correlate only moderately with the patients' experience.

\section{Definitions and method}

\section{Definition of health-related quality of life}

The term "quality of life" is a multidimensional construct of physical, psychological and social aspects. Since the subjectively perceived quality of life can also depend on affluence, freedom, politics, education, culture and religion, the term "health-related quality of life" (HRQOL) is often used, when the influence of health-related factors is to be considered.

\section{INFOBOX}

Health-related quality of life (HRQOL) was defined in 1993 by the WHO [2] as:

"An individual's perception of their position in life in the context of the culture and value systems in which they live and in relation to their goals, expectations, standards and concerns. It is a broad ranging concept affected in a complex way by the person's physical health, psychological state, personal beliefs, social relationships and their relationship to salient features of their environment."
HRQOL is one of the patient-reported outcomes (PROs). Put simply, a PRO is a measure of how patients perceive their own health or quality of life.

\section{INFOBOX}

Patient-Reported Outcome (PRO) was defined by the FDA as: "A measurement based on a report that comes directly from the patient (i. e. the study subject) about the status of the patient's health condition without alteration or interpretation of the patient's response by a clinician or anyone else."

\section{Recording health-related quality of life}

$\mathrm{HRQOL}$ is assessed using validated questionnaires. A distinction is made between (disease-) specific and generic (non-diseasespecific) instruments ( $\triangleright$ Table $\mathbf{1}$ ). The specific instruments are more sensitive with regard to changes in the observed disease and are usually regarded by patients as more relevant than generic instruments, as they reflect their problems better. Generic instruments allow comparisons irrespective of disease and play an important role, for instance in health economic evaluations. The cancer-specific instrument used most often in North America is the FACT questionnaire, and the EORTC QLQ-C30 is used most often in Europe. Both have many additional tumour-, symptom- or treatment-specific modules and have been validated in many languages.

The HRQOL can be assessed globally by a value on a scale. The EORTC QLQ-C30 questionnaire records the global HRQOL as the mean of two subjective self-assessments on a scale from 1 (very bad) to 7 (excellent) using the following questions: 1) "How would you rate your overall health during the past week?" and 2) "How would you rate your overall quality of life during the past week?". Apart from the global assessment of quality of life, various functional areas and symptoms that can have a potential influence on HRQOL are recorded. The EORTC QLQ-C30 also surveys the physical, emotional, social and cognitive function and role function (adverse effects in work or leisure activities) as well as typical 
symptoms during or after a cancer therapy (fatigue, sleep disturbances, pain, shortness of breath, nausea/vomiting, loss of appetite, constipation, diarrhoea) and financial effects.

A new sum score was recently introduced by the EORTC group, which combines and weights the individual functions and symptoms of the EORTC QLQ-C30. This sum score showed better prognostic values for general survival than the global HRQOL score and every other score of the EORTC QLQ-C30 [3].

Breast cancer-specific functions and symptoms are recorded with the EORTC QLQ-BR45 module, for example symptoms in the breast and/or arm/shoulder or sexual activity. The BR45 module is an extension of the older BR23 module.

\section{Reference values}

Comparisons with the general population are helpful for classifying HRQOL in cancer patients. For the EORTC QLQ-C30 there are standardised values from a representative sample $(n=4,684)$ of the German population stratified by age and sex [4]. Reference values for the EORTC QLQ-C30 for breast cancer patients at both the early and the advanced stage were also published recently, stratified according to various criteria (age, region, performance status, comorbidities), which clinicians can use for interpreting their patients' QOL scores [5].

\section{Interpretation and analysis of the assessments}

Standards for collection, statistical analysis and reporting of PROs have recently been produced from the Setting International Standards in Analyzing Patient-Reported Outcomes and Quality of Life Endpoints Data initiative (SISAQOL) (https://event.eortc. org/sisaqol/conferences-and-publications/). As with every measurement, possible inaccuracies or systematic bias must be considered when assessing PROs. A response shift is one possibility.

\section{INFOBOX}

\section{Response shift}

Response shift denotes a change in the evaluation background for subjective surveys in the course of serious diseases. This can occur in the form of reprioritisation or reconceptualisation: values or aims that a subject regarded as important for quality of life may be assessed differently quantitatively or qualitatively after breast cancer than before the disease. This could explain the phenomenon that quality of life after surviving breast cancer treatment is often above the scores before the diagnosis and above the reference scores of the age-matched general population. Response shift can also be due to recalibration, however: patients who, for instance, suffered from very severe pain in the acute treatment phase possibly judge later moderate pain lower on a scale of $0-10$ than they would have assessed the same pain before the acute phase. When quality of life is compared between randomised groups, however, the response shift usually does not play a significant part.

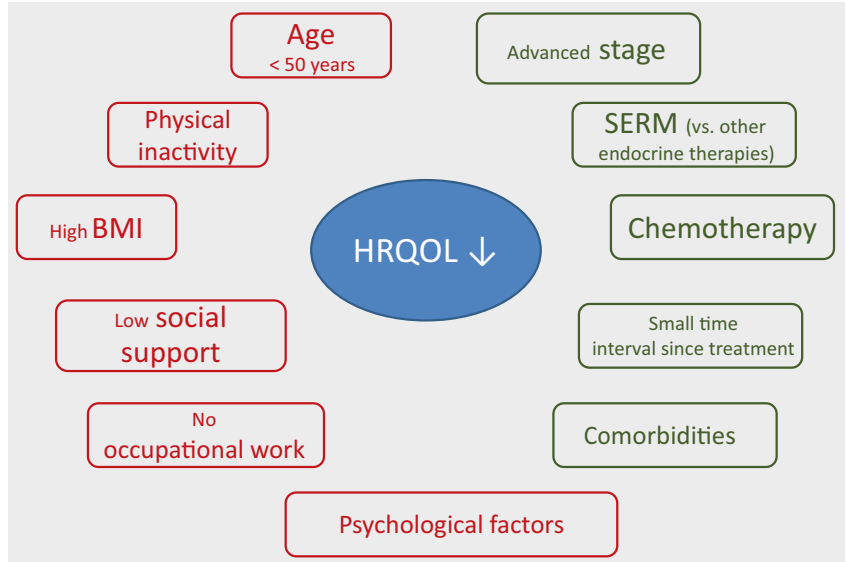

- Fig. 1 Personal (red) and clinical (green) factors associated with reduced health-related quality of life.

\section{Epidemiology}

\section{Determinants and co-factors of health-related quality of life}

HRQOL is generally determined by sociodemographic factors, and also by clinical factors in the case of cancer patients ( $\bullet$ Fig. 1). In the general population, men generally report better HRQOL, higher function scores and fewer symptoms than women. In both sexes, the function scores fall and the symptoms increase in the general population with increasing age [4]. By contrast, lower age is associated with poorer HRQOL or more symptoms in patients with non-metastatic breast cancer [6, 7]. Moreover, studies showed lower HRQOL with high body mass index (BMI) and physical inactivity $[7,8]$. These factors, which can be altered in principle, are therefore a not insignificant starting point for improving quality of life. Low social support and loss of occupational employment are also important factors that should be borne in mind with regard to quality of life. In addition, previous mental problems (previous or existing depressive symptoms, mental illness) are also associated with greater losses of HRQOL and a greater symptom burden, especially fatigue [7, 8]. In a German study of 2671 long-term breast cancer survivors, $17 \%$ reported moderate to major anxiety about recurrence, which was associated with negative effects on quality of life [9]. Apart from relevant clinical aspects as regards combatting the cancer, predictive factors with regard to quality of life after breast cancer should also be considered.

Of the usual breast cancer treatments, chemotherapy has the largest negative effect on HRQOL $[6,8]$. No major differences were shown between chemotherapies with or without taxanes [10]. Patients with early-stage breast cancer who had received selective oestrogen receptor modulators (SERMs) showed more depressive symptoms than patients on other endocrine therapies [6]. The influence of the treatment diminishes with time, however, and no longer plays a major part in longer-term quality of life. Patients with advanced disease report worse HRQOL on average than patients with early disease [6]. Fatigue, pain, mental and cognitive problems appear to be especially relevant for quality of life after breast cancer $[11,12]$. 


\section{Course of health-related quality of life and relevant PROs after breast cancer}

During adjuvant therapy, breast cancer patients on average report significantly worse global quality of life and worse values in all functional areas (physical, emotional, social, cognitive and role function), more symptoms, especially fatigue and sleep disorders, as well as more financial worries than women of the same age in the general population. One or more years after the conclusion of adjuvant breast cancer therapy, (disease-free) women assessed their quality of life on average as equally good or even better than women of comparable age in the general population, but there is still a significantly greater symptom burden [12-14].

- Fig. 2 shows problems reported frequently by women after breast cancer. Several years after the breast cancer diagnosis, patients still report, for example, cognitive impairments such as reduced concentration and memory problems. Cognitive function was still significantly worse in various studies in women even several years after breast cancer than in women of comparable age in the general population [12-15]. The precise reasons for this are still unclear and are currently being further investigated. Furthermore, sleep problems were also significantly more frequent after 5 years in diseasefree breast cancer survivors than in the reference population $[12,13]$.

Especially frequent problems, for which women would like more support after breast cancer, are sexual complaints (e.g. vaginal dryness, dyspareunia, loss of libido) and hot flushes [12, 16]. Menopausal symptoms are not surprising in the patients' age group. However, one study in 843 disease-free women about 6 years after breast cancer diagnosis (without or after conclusion of endocrine therapy) showed significantly worse vasomotor and sexual scores compared with controls from the general population [17].

Fatigue is one of the most frequent problems during adjuvant therapy. The prevalence and intensity decrease markedly in the months and years after the end of treatment. However, roughly a quarter of disease-free women still report moderate to severe fatigue even 5 years after the breast cancer diagnosis [12, 13]. This is an considerable problem, especially because fatigue showed the greatest impairment of HRQOL in different studies and is also one of the main reasons why women do not return to work after breast cancer [15]. Predictors for persistent fatigue include a lack of physical activity, severe overweight, mental problems, sleep problems, lack of social support and also menopausal symptoms [8]. Chemotherapy, on the other hand - one of the most important factors for the occurrence of fatigue - is not a predictor of long-term persistence of fatigue.

The HRQOL can be limited by other treatment-specific side effects such as arthralgia, a common side effect of aromatase inhibitors, or polyneuropathy, a potential side effect of some chemotherapies. In addition, women often have reduced cardiorespiratory fitness even several years after surviving breast cancer.

\section{Measures to improve quality of life after breast cancer}

The development of cancer treatments with fewer side effects can help to reduce the impairment of HRQOL. Side effects cannot be

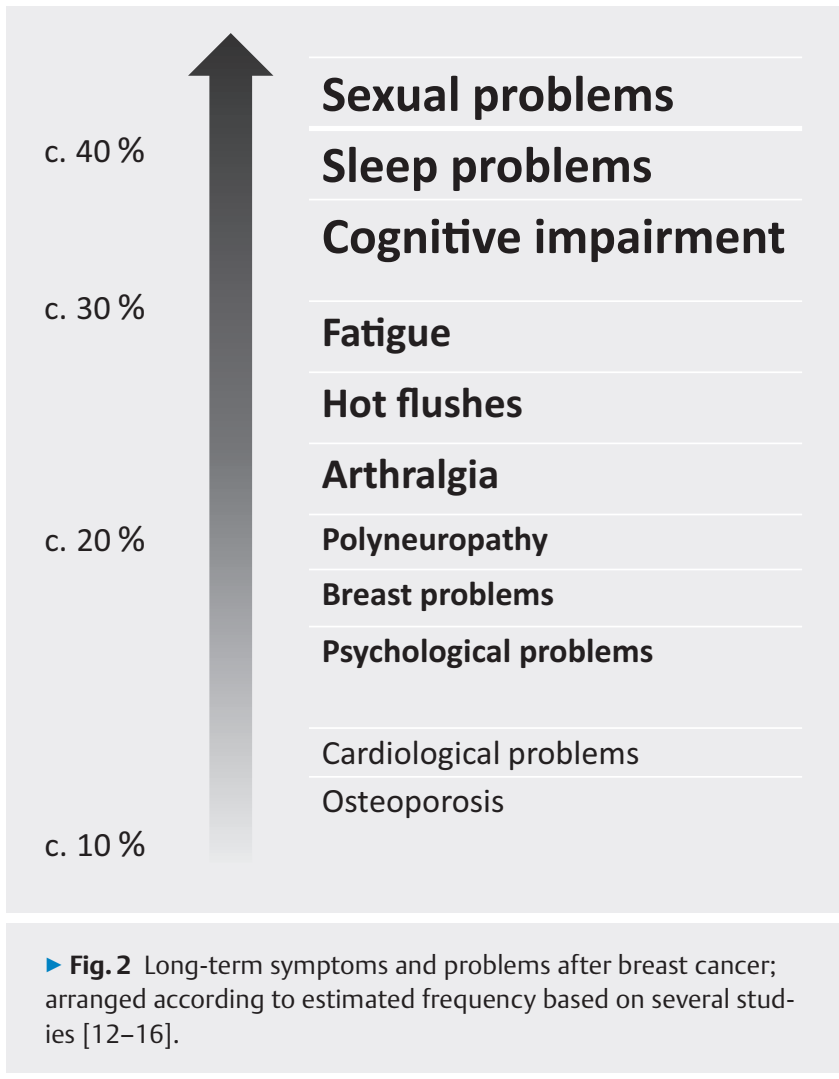

avoided entirely, however, and must be balanced against the efficacy of the treatment. Supportive measures are therefore all the more important for improving or maintaining quality of life. An important first step would be monitoring symptoms by asking patients about them systematically. In practice, however, it appears that fatigue, for example, is addressed too rarely by physicians or nursing staff. Many patients do not report it on their own initiative [15], partly from not knowing that their symptoms are a potentially treatable problem, and partly from a lack of communication skills or simply lack of time when talking with their doctor. In consequence, this symptom, which greatly limits quality of life, is often treated insufficiently. A randomised controlled study by Ethan Basch et al. [18] showed that even simple internet-based monitoring of 12 common symptoms, which sent a notification to the study nurse if there was a deterioration, led to significant improvements in HRQOL in patients with metastatic disease and even to significantly longer survival. In addition to symptom monitoring, consideration of psychosocial factors, such as previous or existing depressive symptoms, anxiety states, social support, financial, familiar or organisational difficulties because of the disease, would be useful so that measures to maintain quality of life could be adopted early if needed, such as arranging contact to a supportive care program.

Moreover, non-pharmacological interventions to maintain or improve HRQOL or to reduce the symptom burden have been investigated in numerous randomised controlled trials ( $\triangleright$ Table 2 ). Currently, there is good evidence for the efficacy of yoga, strength/ endurance training, cognitive behaviour therapy (CBT) and mindfulness-based stress reduction (MBSR) with regard to HRQOL [19- 
- Table 2 Non-pharmacological interventions with well-attested effects on HRQOL.

\begin{tabular}{|l|l|l|l|}
\hline intervention & $\begin{array}{l}\text { good evidence for } \\
\text { effects on: }\end{array}$ & probable ${ }^{1}$ further effects on: & comment \\
\hline $\begin{array}{l}\text { strength/endurance } \\
\text { training [20] }\end{array}$ & $\begin{array}{l}\text { HRQoL, fatigue, physical } \\
\text { fitness, anxiety, depression }\end{array}$ & $\begin{array}{l}\text { sleep, bone density, polyneuropathy, } \\
\text { cognitive function, cardiotoxicity, fall } \\
\text { prevention, pain, sexual function }\end{array}$ & $\begin{array}{l}\text { training recommendations must be } \\
\text { better individualised by further } \\
\text { research. }\end{array}$ \\
\hline Yoga [21] & $\begin{array}{l}\text { HRQoL, fatigue, sleep, } \\
\text { depression, anxiety, pain, physical } \\
\text { fitness, fall prevention, pain }\end{array}$ & $\begin{array}{l}\text { which type of yoga achieves the best } \\
\text { effects must be further investigated. } \\
\text { Tai-chi and Qigong have also shown } \\
\text { some good effects. }\end{array}$ \\
\hline $\begin{array}{l}\text { psychosocial, educational } \\
\text { and/or behaviour-based } \\
\text { interventions } \\
\text { (e. g. MBSR, CBT) 19] }\end{array}$ & partially for fatigue, HRQoL & depending on intervention focus & $\begin{array}{l}\text { interventions specifically focused on } \\
\text { certain symptoms often achieve } \\
\text { better effects for this symptom. }\end{array}$ \\
\hline \begin{tabular}{l} 
1 Evidence moderate or studies as yet insufficient. \\
\hline
\end{tabular} & & & \\
\hline
\end{tabular}

22]. Which intervention works best in a given situation is still unclear. Current studies should match the interventions better to the characteristics of the individual patient, treatment and symptom.

\section{Conflict of Interest}

The authors declare that they have no conflict of interest.

\section{References}

[1] Kluetz PG et al. Focusing on Core Patient-Reported Outcomes in Cancer Clinical Trials-Response. Clin Cancer Res 2016; 22 (22): 5618

[2] Group W. Study protocol for the World Health Organization project to develop a quality of lifeinstrument (WHOQOL). Quality of Life Research 1993; 2: 153-159

[3] Husson O et al. The EORTC QLQ-C30 Summary Score as Prognostic Factor for Survival of Patients with Cancer in the "Real-World": Results from the Population-Based PROFILES Registry. Oncologist 2019; Epub ahead of print

[4] Waldmann A, Schubert D, Katalinic A. Normative data of the EORTC QLQC30 for the German population: a population-based survey. PLoS One 2013; 8 (9): e74149

[5] Mierzynska J et al. Reference values for the EORTC QLQ-C30 in early and metastatic breast cancer. Eur J Cancer 2020; 125: 69-82

[6] Hamer J et al. Quality of life (QOL) and symptom burden (SB) in patients with breast cancer. Support Care Cancer 2017; 25 (2): 409-419

[7] Sousa $\mathrm{H}$ et al. A systematic review of factors affecting quality of life after postmastectomy breast reconstruction in women with breast cancer. Psychooncology 2019; 28 (11): 2107-2118

[8] Schmidt ME et al. Determinants of physical, affective, and cognitive fatigue during breast cancer therapy and 12 months follow-up. Int J Cancer 2018; 142 (6): 1148-1157

[9] Koch $L$ et al. Fear of recurrence in long-term breast cancer survivors-still an issue. Results on prevalence, determinants, and the association with quality of life and depression from the cancer survivorship-a multi-regional population-based study. Psychooncology 2014; 23 (5): 547-554
[10] Willson ML et al. Taxanes for adjuvant treatment of early breast cancer. Cochrane Database Syst Rev 2019; 9: CD004421

[11] Chow $S$ et al. Symptoms Predictive of Overall Quality of Life Using the Edmonton Symptom Assessment Scale in Breast Cancer Patients Receiving Radiotherapy. Clin Breast Cancer 2019; 19 (6): 405-410

[12] Schmidt ME, Wiskemann J, Steindorf K. Quality of life, problems, and needs of disease-free breast cancer survivors 5 years after diagnosis. Qual Life Res 2018; 27(8): 2077-2086

[13] Arndt V et al. Quality of life in long-term and very long-term cancer survivors versus population controls in Germany. Acta Oncol 2017; 56 (2): 190-197

[14] Hsu T et al. Quality of life in long-term breast cancer survivors. J Clin Oncol 2013; 31 (28): 3540-3548

[15] de Ligt KM et al. The impact of health symptoms on health-related quality of life in early-stage breast cancer survivors. Breast Cancer Res Treat 2019; 178 (3): 703-711

[16] Bloom JR, Petersen DM, Kang SH. Multi-dimensional quality of life among long-term ( $5+$ years) adult cancer survivors. Psychooncology 2007; 16 (8): 691-706

[17] Davis SR et al. Menopausal symptoms in breast cancer survivors nearly 6 years after diagnosis. Menopause 2014; 21 (10): 1075-1081

[18] Basch E et al. Symptom Monitoring With Patient-Reported Outcomes During Routine Cancer Treatment: A Randomized Controlled Trial. ] Clin Oncol 2016; 34 (6): 557-565

[19] Duncan M et al. Review of systematic reviews of non-pharmacological interventions to improve quality of life in cancer survivors. BMJ Open 2017; 7 (11): e015860

[20] Campbell KL et al. Exercise Guidelines for Cancer Survivors: Consensus Statement from International Multidisciplinary Roundtable. Med Sci Sports Exerc 2019; 51 (11): 2375-2390

[21] Danhauer SC et al. Yoga for symptom management in oncology: A review of the evidence base and future directions for research. Cancer 2019; 125 (12): 1979-1989

[22] Matsuda A et al. Effectiveness of psychoeducational support on quality of life in early-stage breast cancer patients: a systematic review and metaanalysis of randomized controlled trials. Qual Life Res 2014; 23 (1): 21-30 